\title{
REGULARITY AND LINEARITY DEFECT OF MODULES OVER LOCAL RINGS
}

\author{
RASOUL AHANGARI MALEKI AND MARIA EVELINA ROSSI
}

\begin{abstract}
Given a finitely generated module $M$ over a commutative local ring (or a standard graded $k$-algebra) $(R, \mathfrak{m}, k)$, we detect its complexity in terms of numerical invariants coming from suitable $\mathfrak{m}$-stable filtrations $\mathbb{M}$ on $M$. We study the Castelnuovo-Mumford regularity of $\operatorname{gr}_{\mathbb{M}}(M)$ and the linearity defect of $M$, denoted $\operatorname{ld}_{R}(M)$, through a deep investigation based on the theory of standard bases. If $M$ is a graded $R$-module, then $\operatorname{reg}_{R}\left(g r_{\mathbb{M}}(M)\right)<\infty$ implies $\operatorname{reg}_{R}(M)<\infty$ and the converse holds provided $M$ is of homogenous type. An analogous result can be proved in the local case in terms of the linearity defect. Motivated by a positive answer in the graded case, we present for local rings a partial answer to a question raised by Herzog and Iyengar of whether $\operatorname{ld}_{R}(k)<\infty$ implies $R$ is Koszul.
\end{abstract}

Introduction and notation. Throughout this paper, $(R, \mathfrak{m}, k)$ is a commutative Noetherian local ring (or a standard graded $k$-algebra) with maximal ideal (or homogeneous maximal ideal) $\mathfrak{m}$ and residue field $k$. All the modules we consider are finitely generated over $R$. Let $M$ be an $R$-module, according to [12]. We say that a descending filtration of submodules $\mathbb{M}=\left\{\mathfrak{F}_{p} M\right\}_{p \geq 0}$ of $M=\mathfrak{F}_{0} M$ is an $\mathfrak{m}$-filtration if $\mathfrak{m} \mathfrak{F}_{p} M \subseteq \mathfrak{F}_{p+1} M$ for every $p \geq 0$, and $\mathbb{M}$ is an $\mathfrak{m}$-stable (or good) filtration if $\mathfrak{m} \mathfrak{F}_{p} M=\mathfrak{F}_{p+1} M$ for all sufficiently large $p$. In the following, a filtered module $M$ will always be an $R$-module equipped with an $\mathfrak{m}$ -

2010 AMS Mathematics subject classification. Primary 16W50, 13D07, Secondary $16 \mathrm{~W} 70,16 \mathrm{~S} 37$.

Keywords and phrases. Regularity, linearity defect, minimal free resolutions, standard basis, associated graded module, filtered modules, Koszul algebras.

The first author was in part supported by a grant from IPM (No. 92130028). The second author was supported by MIUR, PRIN 2010-11 (GVA). This work was partly accomplished while the first author was visiting the University of Genoa.

Received by the editors on June 6, 2013, and in revised form on September 4, 2013. 
stable filtration $\mathbb{M}$. Define

$$
g r_{\mathbb{M}}(M)=\bigoplus_{p \geq 0}\left(\mathfrak{F}_{p} M / \mathfrak{F}_{p+1} M\right)
$$

to be the associated graded module to $M$ with respect to the filtration $\mathbb{M}$. If $\mathbb{M}=\left\{\mathfrak{m}^{p} M\right\}$ is the $\mathfrak{m}$-adic filtration on $M$, we denote $\operatorname{gr}_{\mathbb{M}}(M)$ simply by $M^{g}$. Then $g r_{\mathbb{M}}(M)$ is a graded $R^{g}$-module. When $R$ is a standard graded $k$-algebra, then $R^{g}$ is naturally isomorphic to $R$.

The main goal of this paper is to study properties of the module $M$, such as the linearity defect, Koszulness and regularity by means of the graded structure of $\operatorname{gr}_{\mathbb{M}}(M)$ for a given $\mathfrak{m}$-stable filtration $\mathbb{M}$. The strength of our approach comes from a deep investigation of the interplay between a minimal free resolution of $g r_{\mathbb{M}}(M)$ as an $R^{g}$-module and a free resolution of $M$ as an $R$-module coming from the theory of standard bases.

To avoid triviality, we assume that $g r_{\mathbb{M}}(M)$ is not zero or equivalently $M \neq 0$. We consider a minimal free resolution of $M$ as a finitely generated $R$-module, that is, a complex of free $R$-modules

$$
\mathfrak{F}: \cdots \rightarrow F_{i+1} \stackrel{\phi_{i+1}}{\longrightarrow} F_{i} \stackrel{\phi_{i}}{\longrightarrow} F_{i-1} \rightarrow \cdots \rightarrow F_{1} \stackrel{\phi_{1}}{\longrightarrow} F_{0} \rightarrow 0
$$

such that $\mathrm{H}_{i}(\mathfrak{F})=0$ for $i>0$ and $\mathrm{H}_{0}(\mathfrak{F})=M, \phi_{i+1}\left(F_{i+1}\right) \subseteq \mathfrak{m} F_{i}$ for every $i$. Such a resolution exists and it is unique up to an isomorphism of complexes. By definition, the $i$ th Betti number $\beta_{i}^{R}(M)$ of $M$ is the rank of $F_{i}$, that is, $\beta_{i}^{R}(M)=\operatorname{dim}_{k} \operatorname{Tor}_{i}^{R}(M, k)$. It is well known that, given a minimal graded free resolution $\mathbf{G}$ of $\operatorname{gr}_{\mathbb{M}}(M)$ as $R^{g}$-module, one can build up a free resolution $\mathfrak{F}$ of $M$ (not necessarily minimal) equipped with a special filtration $\mathbb{F}$ on $\mathfrak{F}$ such that $g r_{\mathbb{F}}(\mathbf{F})=\mathbf{G}$ (see $[\mathbf{1 4}$, 2.4]). In some cases, the process for obtaining a minimal free resolution of $M$ starting from $g r_{\mathbb{M}}(M)$ is under control via special cancelations (see $[\mathbf{1 0}, 3.1])$. As a consequence of this construction

$$
\beta_{i}^{R}(M) \leq \beta_{i}^{R^{g}}\left(g r_{\mathbb{M}}(M)\right)
$$

and $M$ is said of homogeneous type with respect to $\mathbb{M}$ if the equality holds for every $i \geq 0$. This is equivalent to say that the resolution $\mathbb{F}$ in the above construction is minimal. Interesting examples of modules of homogeneous type are given in literature, see for example, [7] or [10, $1.10,1.12]$. It is well known that, if $g r_{\mathbb{M}}(M)$ has a linear resolution, then $M$ is of homogeneous type. 
If $R$ is a standard graded $k$-algebra and $M$ is a finitely generated graded $R$-module, then $\operatorname{Tor}_{i}^{R}(M, k)$ inherits the graded structure and the $(i, j)$-th graded Betti number $\beta_{i j}^{R}(M)=\operatorname{dim}_{k} \operatorname{Tor}_{i}^{R}(M, k)_{j}$ of $M$ is the number of copies of $R(-j)$ that appear in $F_{i}$ (recall $R(-j)_{i}:=$ $\left.R_{i-j}\right)$. We set

$$
t_{i}^{R}(M)=\sup \left\{j: \beta_{i j}^{R}(M) \neq 0\right\}
$$

where, by convention, $t_{i}^{R}(M)=-\infty$ if $F_{i}=0$. By definition, $t_{0}^{R}(M)$ is the largest degree of a minimal generator of $M$. An important invariant associated to a minimal free resolution of $M$ as an $R$-module is the Castelnuovo-Mumford regularity

$$
\operatorname{reg}_{R}(M)=\sup \left\{j-i: \beta_{i j}^{R}(M) \neq 0\right\}=\sup \left\{t_{i}^{R}(M)-i: i \in \mathbf{N}\right\} .
$$

It is clear that $\operatorname{reg}_{R}(M)$ can be infinite. In the graded case we have a double opportunity: we can take advantage both of the graded $R$ structure of $M$ and of the graded $R$-structure of $g r_{\mathbb{M}}(M)$. We present explicit bounds on the graded Betti numbers of $M$ in terms of the degrees of a minimal system of generators of $M$ and of the Betti numbers of $g r_{\mathbb{M}}(M)$. In particular, we prove that if there exists a filtration $\mathbb{M}$ of $M$ such that $\operatorname{reg}_{R}\left(g r_{\mathbb{M}}(M)\right)<\infty$, then $\operatorname{reg}_{R}(M)<\infty$. If $M$ is of homogeneous type with respect to $\mathbb{M}$, then also the converse holds, see Corollary 2.6. Example 2.7 shows that the assumption is necessary.

If $R$ is graded, the residue field $k$ has a special behavior. Avramov and Peeva in [4] proved that either $k$ admits a linear resolution, that is, $\operatorname{reg}_{R}(k)=0$, or $\operatorname{reg}_{R}(k)$ is infinite. Following the classical definition given by Priddy, if $k$ admits a linear resolution, we say that $R$ is Koszul.

For local rings we say that $R$ is Koszul if and only if $R^{g}$ is Koszul. One can reformulate the analogous of the statement proved by Avramov and Peeva for local rings replacing the concept of regularity with a new invariant that can be defined for any finitely generated module $M$ over a local ring. This invariant is the linearity defect, denoted $\operatorname{ld}_{R}(M)$. This notion was introduced by Herzog and Iyengar in [6] and studied further by Iyengar and Römer in [8], see also Section 3. The linearity defect measures how far $M^{g}$ is from having a linear resolution. Notice that if $\operatorname{ld}_{R}(M)<\infty$, then the Poincaré series $P_{M}(t)=\sum_{i \geq 0} \beta_{i}(M) t^{i}$ of $M$ is rational sharing all modules a common denominator depending only on $R^{g}$, see $[6,1.8]$. 
In $[6,1.14]$ the following challenging question has been stated.

Question 0.1. If $\operatorname{ld}_{R}(k)<\infty$, does it follow that $\operatorname{ld}_{R}(k)=0$ ?

In the graded case $\operatorname{ld}_{R}(k)<\infty$ implies $\operatorname{reg}_{R}(k)<\infty$. Then $R$ is Koszul as mentioned before by the results of Avramov and Peeva. In the local case, the problem is still open, and it is one of the aims of our investigation. Interesting answers are given in a recent paper of Şega for certain classes of local rings, see [13].

The above question is equivalent to proving that $\operatorname{ld}_{R}(k)<\infty$ implies $\operatorname{ld}_{R^{g}}(k)<\infty$. In analogy, since $k=k^{g}$, a more general question arises:

Question 0.2. Let $M$ be a finitely generated $R$-module. If $\operatorname{ld}_{R}(M)<$ $\infty$, does it follow that $\operatorname{ld}_{R^{g}}\left(M^{g}\right)<\infty$ ?

In general the answer to Question 0.2 is negative; nevertheless, this does not disprove Question 0.1. For instance, Example 2.7 presents a graded module of finite projective dimension, hence of finite linearity defect; but the regularity of $M^{g}$ is infinite, hence the linearity defect of $M^{g}$ is infinite by $[6,1.2]$. In view of giving an answer to Question 0.1, since $k$ is a cyclic $R$-module, it could be interesting to analyze also Example 4.1 which provides a cyclic module with same features.

In Proposition 3.5, we prove that if $\operatorname{ld}_{R}(M)=d<\infty$, then

$$
\operatorname{reg}_{R}(M)=\max \left\{t_{i}(M)-i: 0 \leq i \leq d\right\}
$$

as an expected specification of $[6,1.9]$, where it is proved that, if $\operatorname{ld}_{R}(M)<\infty$, then $\operatorname{reg}_{R}(M)<\infty$. In particular, if $M$ is a Koszul $R$ module, then $\operatorname{reg}_{R}(M)=t_{0}(M)$. Theorem 4.4 presents the analogous result in the local case. We prove that, if $M$ is a module of homogeneous type with respect to the filtration $\mathbb{M}$, then $\operatorname{ld}_{R}(M)=d<\infty$ implies $\operatorname{reg}_{R^{g}}\left(g r_{\mathbb{M}}(M)\right)=\max \left\{t_{i}\left(g r_{\mathbb{M}}(M)\right)-i: 0 \leq i \leq d\right\}$. This allows us to present a positive answer to Question 1 when $k$ is of homogeneous type with respect to a stable filtration $\mathbb{M}$, see Proposition 4.5.

1. Preliminaries on filtered modules. We refer to $[\mathbf{1 0}, \mathbf{1 2}]$ for more facts concerning filtered modules and filtered complexes. We 
include in this section the results, and we fix the definitions that will be used in the present paper.

If $N$ is a submodule of an $R$-module $M$, by the Artin-Rees lemma, the collection $\left\{N \cap \mathfrak{F}_{p} M \mid p \geq 0\right\}$ is an $\mathfrak{m}$-filtration of $N$, and we denote $g r_{\mathbb{M}}(N)$ the associated graded module induced by $\mathbb{M}$ on $N$. Since

$$
\left(N \cap \mathfrak{F}_{p} M\right) /\left(N \cap \mathfrak{F}_{p+1} M\right) \cong\left(N \cap \mathfrak{F}_{p} M+\mathfrak{F}_{p+1} M\right) / \mathfrak{F}_{p+1} M,
$$

$g r_{\mathbb{M}}(N)$ is a graded submodule of $g r_{\mathbb{M}}(M)$. If $x \in M \backslash 0$, we denote by $v_{\mathbb{M}}(x)$ the largest integer $p$ such that $x \in \mathfrak{F}_{p} M$ (the so-called valuation of $x$ with respect to $\mathbb{M})$ and we denote by $x^{*}$ or $g r_{\mathbb{M}}(x)$ the residue class of $x$ in $\mathfrak{F}_{p} M / \mathfrak{F}_{p+1} M$ where $p=v_{\mathbb{M}}(x)$. If $x=0$, we set $v_{\mathbb{M}}(x)=+\infty$.

Using (1), it is clear that

$$
g r_{\mathbb{M}}(N)=\left\langle g r_{\mathbb{M}}(x): x \in N\right\rangle
$$

Definition 1.1. Let $M$ be a filtered $R$-module. A subset $S=$ $\left\{f_{1}, \ldots, f_{s}\right\}$ of $M$ is called a standard basis of $M$ with respect to $\mathbb{M}$ if

$$
g r_{\mathbb{M}}(M)=\left\langle g r_{\mathbb{M}}\left(f_{1}\right), \cdots, g r_{\mathbb{M}}\left(f_{s}\right)\right\rangle .
$$

If any proper subset of $S$ is not a standard basis of $M$, we call $S$ a minimal standard basis.

We remark that the number of generators and the corresponding valuations of a minimal standard basis depend only on $M$ and $\mathbb{M}$.

Definition 1.2. Let $M$ and $N$ be filtered $R$-modules, and let $f$ : $M \rightarrow N$ be a homomorphism of $R$-modules. Then $f$ is said to be a homomorphism of filtered modules if $f\left(\mathfrak{F}_{p} M\right) \subseteq \mathfrak{F}_{p} N$ for every $p \geq 0$ and $f$ is said to be strict if $f\left(\mathfrak{F}_{p} M\right)=f(M) \cap \mathfrak{F}_{p} N$ for every $p \geq 0$.

Let $F=\bigoplus_{i=1}^{s} R e_{i}$ be a free $R$-module of rank $s$, and let $v_{1}, \ldots, v_{s}$ be integers. We define the filtration $\mathbb{F}=\left\{\mathfrak{F}_{p} F: p \in \mathbf{Z}\right\}$ on $F$ as follows:

$$
\mathfrak{F}_{p} F:=\bigoplus_{i=1}^{s} \mathfrak{m}^{p-v_{i}} e_{i}=\left\{\left(a_{1}, \cdots, a_{s}\right): a_{i} \in \mathfrak{m}^{p-v_{i}}\right\}
$$


If $i \leq 0$ we set $\mathfrak{m}^{i}=R$. We denote the filtered free $R$-module $F$ by $\bigoplus_{i=1}^{s} R v_{i}$, and we call it special filtration on $F$. It is clear that the filtration $\mathbb{F}$ defined above is an $\mathfrak{m}$-stable filtration.

If $(\mathbf{F}, d$. $)$ is a complex of finitely generated free $R$-modules, $\left(d_{i}\right.$ : $F_{i} \rightarrow F_{i-1}$ denotes the $i$-th differential map), a special filtration on $\mathbf{F}$ is a special filtration on each $F_{i}$ that makes $(\mathbf{F}, d$.) a filtered complex.

Let $M$ be an $R$-module equipped with the filtration $\mathbb{M}$, and let $S=\left\{f_{1}, \ldots, f_{s}\right\}$ be a system of elements of $M$ with $v_{\mathbb{M}}\left(f_{i}\right)$ the corresponding valuations. Let $F=\bigoplus_{i=1}^{s} R e_{i}$ be a free $R$-module of rank $s$ equipped with the filtration $\mathbb{F}$ where $v_{i}=v_{\mathbb{M}}\left(f_{i}\right)$. Then we denote the filtered free $R$-module $F$ by $\bigoplus_{i=1}^{s} R v_{\mathbb{M}}\left(f_{i}\right)$, and hence $v_{\mathbb{F}}\left(e_{i}\right)=v_{\mathbb{M}}\left(f_{i}\right)$.

Let $d: F \rightarrow M$ be a morphism of filtered $R$-modules defined by

$$
d\left(e_{j}\right)=f_{j} .
$$

It is clear that $d$ is a morphism of filtered $R$-modules and $g r_{\mathbb{F}}(F)$ is isomorphic to the graded free $R^{g}$-module $\bigoplus_{i=1}^{s} R^{g}\left(-v_{\mathbb{M}}\left(f_{i}\right)\right)$ with a basis $\left(e_{1}, \ldots, e_{s}\right)$ where $\operatorname{deg}\left(e_{i}\right)=v_{\mathbb{M}}\left(f_{i}\right)$. In particular, $d$ induces a natural graded morphism (of degree zero) $g r(d): g r_{\mathbb{F}}(F) \rightarrow g r_{\mathbb{M}}(M)$ sending $e_{j}$ to $g r_{\mathbb{M}}\left(f_{j}\right)$.

In particular, $\operatorname{gr}\left({ }^{*}\right)$ is a functor from the category of the filtered $R$-modules into the category of the graded $R^{g}$-modules.

Let $c={ }^{t}\left(c_{1}, \ldots, c_{s}\right)$ be an element of $F$ and ${ }^{t}(\quad)$ denotes the transposed vector. By the definition of the filtration $\mathbb{F}$ on $F$, we have

$$
v_{\mathbb{F}}(c)=\min \left\{v_{R}\left(c_{i}\right)+v_{\mathbb{M}}\left(f_{i}\right)\right\} \leq v_{\mathbb{M}}(d(c))
$$

Set $\operatorname{gr}_{\mathbb{F}}(c)=^{t}\left(c_{1}^{\prime}, \ldots, c_{s}^{\prime}\right)$ and $v=v_{\mathbb{F}}(c)$, then

$$
c_{i}^{\prime}=\left\{\begin{array}{ll}
g r_{\mathfrak{m}}\left(c_{i}\right) & \text { if } v_{R}\left(c_{i}\right)+v_{\mathbb{M}}\left(f_{i}\right)=v \\
0 & \text { if } v_{R}\left(c_{i}\right)+v_{\mathbb{M}}\left(f_{i}\right)>v
\end{array} .\right.
$$

We have a canonical embedding $g r_{\mathbb{F}}(\operatorname{ker}(d)) \rightarrow \operatorname{ker}(g r(d))$. If $\operatorname{gr}(d)$ is surjective, then $d$ is a strict surjective homomorphism, equivalently,

$$
g r_{\mathbb{F}}(\operatorname{ker}(d))=\operatorname{ker}(g r(d)) .
$$


With the previous notation, extending a result of Robbiano and Valla in [9], Shibuta characterized the standard bases of a filtered module (see [15]).

Theorem 1.3. Let $M$ be a filtered $R$-module and $\left\{f_{1}, \cdots, f_{s}\right\}$ elements of $M$. The following facts are equivalent:

(i) $\left\{f_{1}, \ldots, f_{s}\right\}$ is a standard basis of $M$ with respect to $\mathbb{M}$.

(ii) $\left\{f_{1}, \ldots, f_{s}\right\}$ generates $M$ and $d$ is strict.

(iii) $\left\{f_{1}, \ldots, f_{s}\right\}$ generates $M$ and $g r_{\mathbb{F}}(\operatorname{ker}(d))=\operatorname{ker}(g r(d))$.

2. Regularity of filtered graded modules. Let $R$ be a standard graded algebra over a field $k$ with homogeneous maximal ideal $\mathfrak{m}$, and let $M$ be a filtered graded $R$-module, that is, a graded module $M=$ $\oplus_{i \geq 0} M_{i}$ equipped with an $\mathfrak{m}$-stable filtration $\mathbb{M}$. In this section, we will present a comparison between the regularity of $M$ and the regularity of $g r_{\mathbb{M}}(M)$. The approach is elementary and our investigation is mainly based on the theory of standard bases.

In the graded module $M$, we say that an element $x$ is homogeneous if $x \in M_{i}$ for some $i$.

Definition 2.1. Let $M$ be a filtered graded $R$-module, and let $S=$ $\left\{f_{1}, \ldots, f_{s}\right\}$ be a standard basis of $M . S$ is a homogeneous standard basis of $M$ if $f_{i}$ is a homogeneous element of $M$ for every $1 \leq i \leq s$.

The filtration $\mathbb{M}=\left\{\mathfrak{F}_{p} M\right\}_{p \geq 0}$ is a graded filtration if $\mathfrak{F}_{p} M$ is a graded submodule of $M$ for each $p \geq 0$.

For example, the $\mathfrak{m}$-adic filtration $\left\{\mathfrak{m}^{p} M\right\}_{p>0}$ is a graded filtration. When $\mathbb{M}$ is a graded filtration on $M$, then $M$ admits a homogeneous standard basis. In general, a filtered graded module does not necessarily have a homogeneous standard basis. For instance, let $k$ be a field, set $R=k[X, Y] /\left(X^{3}, Y^{4}\right)$, and write $x$ and $y$ for the residue class of $X$ and $Y$ in $R$. Set $\mathfrak{m}=(x, y)$ and $I=\mathfrak{F}_{0} I=\left(x^{2}, y^{3}\right), \mathfrak{F}_{1} I=\left(y^{3}+x^{2}\right)$, $\mathfrak{F}_{p} I=\mathfrak{m} F_{p-1} I$ for $p \geq 2$. Note that $\mathfrak{m} \mathfrak{F}_{0} I=\left(x y^{3}, x^{2} y\right)$, and we have $\mathfrak{m} \mathfrak{F}_{0} I \subset \mathfrak{F}_{1} I$. So $\mathbb{I}=\left\{\mathfrak{F}_{p} I\right\}_{p \geq 0}$ is an $\mathfrak{m}$-stable filtration on $I$. In this case, $I$ does not have a homogeneous standard basis with respect to $\mathbb{I}$. 
From now on, if $M$ is a graded module, a filtration on $M$ will always be a graded filtration.

Let $N$ be a graded $R$-module equipped with the filtration $\mathbb{N}=$ $\left\{\mathfrak{F}_{p} N\right\}_{p \geq 0}$. For every non-zero homogeneous element $x \in N$, we have two integers attached to $x$. We say that $x$ has degree $i$, we write $\operatorname{deg}(x)=i$ if $x \in N_{i}$, and we say that $x$ has valuation $p=v_{\mathbb{N}}(x)$ if $x \in \mathfrak{F}_{p} N \backslash \mathfrak{F}_{p+1} N$.

If $\left\{x_{1}, \ldots, x_{n}\right\}$ is a minimal homogeneous generating set of $N$, we denote by $\mathcal{D}(N)$ the set $\left\{\operatorname{deg}\left(x_{i}\right): 0 \leq i \leq n\right\}$. This set is uniquely determined by $N$. If $N=(0)$, we set $\mathcal{D}(N)=\varnothing$. Let $\left\{f_{1}, \ldots, f_{s}\right\}$ be a minimal homogeneous standard basis of $N$ with respect to $\mathbb{N}$. We set:

$$
\Delta_{\mathbb{N}}(N)=\left\{\operatorname{deg}\left(f_{j}\right)-v_{\mathbb{N}}\left(f_{j}\right): 1 \leq j \leq s\right\}
$$

and

$$
\begin{aligned}
& v_{\mathbb{N}}(N)=\max \Delta_{\mathbb{N}}(N) \\
& u_{\mathbb{N}}(N)=\min \Delta_{\mathbb{N}}(N)
\end{aligned}
$$

When $\mathbb{N}$ is the $\mathfrak{m}$-adic filtration, then $\left\{f_{1}, \ldots, f_{s}\right\}$ is also a minimal homogeneous generating set of $N$, and we have $v_{\mathbb{N}}\left(f_{j}\right)=0$. Hence, one has

$$
\Delta_{\mathbb{N}}(N)=\mathcal{D}(N)
$$

and

$$
v_{\mathbb{N}}(N)=t_{0}(N), \quad u_{\mathbb{N}}(N)=\operatorname{indeg}(N)
$$

The following result completes a well-known comparison between the numerical invariants of a graded free resolution of $\operatorname{gr}_{\mathbb{M}}(M)$ as $R^{g}$. module and a free resolution of $M$ as $R$-module. The local version of the first part of the proposition holds for any commutative ring $R$ (see $[14,2.4])$; we insert here a refinement in the case of graded modules.

Proposition 2.2. Let $R$ be a standard graded $k$-algebra, and let $M$ be a filtered graded $R$-module equipped with an $\mathfrak{m}$-stable graded filtration $\mathbb{M}$. Then there exists a graded free resolution $\mathbf{F}$ of $M$ with a special filtration $\mathbb{F}$ on it such that $\mathbf{G}:=g r_{\mathbb{F}}(\mathbf{F})$ is a minimal graded free resolution of $g r_{\mathbb{M}}(M)$ as an $R$-module. 
Denote by $d_{i}: F_{i} \rightarrow F_{i-1}$ (respectively, $\delta_{i}: G_{i} \rightarrow G_{i-1}$ ) the differential maps of $\mathbf{F}$ (respectively, $\mathbf{G}$ ) and by $\mathbb{F}_{i}$ the special filtration on $F_{i}$. Then, for all $i \geq 0$, we have:

(i) $g r_{\mathbb{F}_{i}}\left(d_{i}\right)=\delta_{i}$.

(ii) $\operatorname{ker}\left(d_{i}\right)$ admits a homogeneous standard basis with respect to $\mathbb{F}_{i}$.

(iii) $\operatorname{ker}\left(\delta_{i}\right)=g r_{\mathbb{F}_{i}}\left(\operatorname{ker}\left(d_{i}\right)\right)$.

(iv) $\Delta_{\mathbb{F}_{i}}\left(\operatorname{ker}\left(d_{i}\right)\right) \subseteq \Delta_{\mathbb{M}}(M)$.

Proof. Let $f_{1}, \ldots, f_{\beta_{0}}$ be a minimal homogeneous standard basis of $M$ with respect to the filtration $\mathbb{M}$. Set $a_{0 j}^{\prime}:=\operatorname{deg}\left(f_{j}\right)$ and $a_{0 j}:=v_{\mathbb{M}}\left(f_{j}\right)$. Then $\Delta_{\mathbb{M}}(M)=\left\{a_{0 j}^{\prime}-a_{0 j}: j=1, \ldots, \beta_{0}\right\}$.

Define $F_{0}=\bigoplus_{j=1}^{\beta_{0}} R\left(-a_{0 j}^{\prime}\right)$ to be the graded $R$-free module of rank $\beta_{0}$ equipped with the special filtration $\mathbb{F}_{0}$ with respect to the integers $v_{\mathbb{M}}\left(f_{1}\right), \cdots, v_{\mathbb{M}}\left(f_{\beta_{0}}\right)$ (as defined in $\left.(2)\right)$. Hence, $v_{\mathbb{F}}\left(e_{0 j}\right)=v_{\mathbb{M}}\left(f_{j}\right)$. We define

$$
d_{0}: F_{0} \rightarrow M
$$

to be the homogeneous homomorphism such that $d_{0}\left(e_{0 j}\right)=f_{j}$. We remark that $R^{g}=R$, and we define

$$
\delta_{0}: G_{0}=\bigoplus_{j=1}^{\beta_{0}} R\left(-a_{0 j}\right) \rightarrow g r_{\mathbb{M}}(M)
$$

with $\delta_{0}\left(e_{0 j}\right)=g r_{\mathbb{M}}\left(f_{j}\right)$. We have the following diagram:

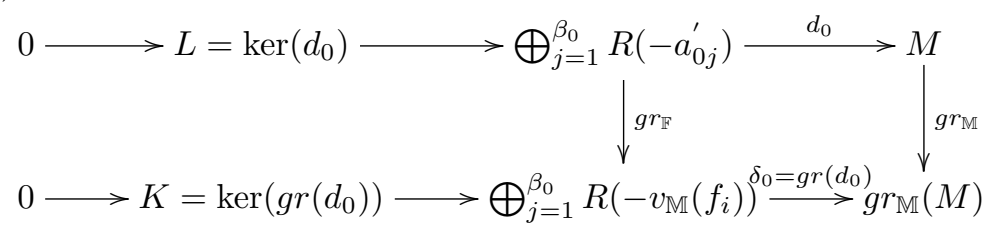

Since $\left\{f_{1}, \ldots, f_{\beta_{0}}\right\}$ is a standard basis of $M$, it generates $M$. Hence, $d_{0}$ is surjective and strict. In particular, by Theorem 1.3, $g r_{\mathbb{F}_{0}}\left(\operatorname{ker}\left(d_{0}\right)\right)=\operatorname{ker}\left(\delta_{0}\right)$.

The special filtration $\mathbb{F}_{0}$ is a graded filtration on the graded free $R$-module $F_{0}$. Since $d_{0}$ is homogeneous, then $L=\operatorname{ker}\left(d_{0}\right)$ is a graded submodule of $F_{0}$, and the induced filtration on $L$, that is $\mathbb{F}_{0} \cap L$ is 
a graded filtration. Therefore, $L=\operatorname{ker}\left(d_{0}\right)$ admits a homogeneous standard basis with respect to the induced filtration. Note that $g r_{\mathbb{F}_{0}}\left(e_{0 j}\right)=e_{0 j}$ and we may write

$$
\operatorname{deg}\left(f_{j}\right)=a_{0 j}^{\prime}=a_{0 j}+c
$$

with $c=\operatorname{deg}\left(f_{j}\right)-v_{\mathbb{M}}\left(f_{j}\right) \in \Delta_{\mathbb{M}}(M)$. Hence, (i), (ii) and (iii) are satisfied for $i=0$. To prove (iv), let $\left\{h_{1}, \ldots, h_{\beta_{1}}\right\}$ be a minimal homogeneous standard basis of $\operatorname{ker}\left(d_{0}\right)$. Assume that $g r_{\mathbb{F}_{0}}\left(h_{i}\right)=$ $\left(x_{i 1}, \ldots, x_{i \beta_{0}}\right)$. There exists a $j$ such that $x_{i j} \neq 0$, so

$$
\operatorname{deg}\left(g r_{\mathbb{F}_{0}}\left(h_{i}\right)\right)=\operatorname{deg}\left(x_{i j}\right)+a_{0 j} .
$$

Let $h_{i}=\left(y_{i 1}, \ldots, y_{i \beta_{0}}\right)$. Since $h_{i}$ is a homogeneous element of $\operatorname{ker}\left(d_{0}\right)$ by (4), $x_{i j}=y_{i j}$, and then we have

$$
\operatorname{deg}\left(h_{i}\right)=\operatorname{deg}\left(y_{i j}\right)+a_{0 j}^{\prime}=\operatorname{deg}\left(g r_{\mathbb{F}_{0}}\left(h_{i}\right)\right)+c,
$$

for some $c \in \Delta_{\mathbb{M}}(M)$. Hence, $\Delta_{\mathbb{F}_{0}}\left(\operatorname{ker}\left(d_{0}\right)\right) \subseteq \Delta_{\mathbb{M}}(M)$.

We prove the result by inductive steps on $n>0$. Assume we have defined filtered graded free modules $F_{0}, \ldots, F_{n-1}$ with special filtration $\mathbb{F}_{0}, \ldots, \mathbb{F}_{n-1}$ such that

$$
F_{n-1} \stackrel{d_{n-1}}{\longrightarrow} F_{n-2} \rightarrow \cdots \rightarrow F_{0} \stackrel{d_{0}}{\longrightarrow} M \rightarrow 0
$$

is a part of a graded free resolution of $M$ and for every $i<n$ we assume:

(i) $g r_{\mathbb{F}_{i}}\left(F_{i}\right)=G_{i}, g r_{\mathbb{F}_{i}}\left(d_{i}\right)=\delta_{i}$

(ii) $\operatorname{ker}\left(d_{i}\right)$ has a homogeneous standard basis with respect to $\mathbb{F}_{i}$,

(iii) $\operatorname{ker}\left(\delta_{i}\right)=g r_{\mathbb{F}_{i}}\left(\operatorname{ker}\left(d_{i}\right)\right)$.

(iv) $\Delta_{\mathbb{F}_{i-1}}\left(\operatorname{ker}\left(d_{i-1}\right)\right) \subseteq \Delta_{\mathbb{M}}(M)$.

Let $\left\{g_{1}, \ldots, g_{\beta_{n}}\right\}$ be a minimal homogeneous standard basis of $\operatorname{ker}\left(d_{n-1}\right)$. We know that $\operatorname{ker}\left(\delta_{n-1}\right)=g r_{\mathbb{F}_{n-1}}\left(\operatorname{ker}\left(d_{n-1}\right)\right)=\left\langle g r_{\mathbb{F}_{n-1}}\left(g_{1}\right)\right.$, $\left.\ldots, g r_{\mathbb{F}_{n-1}}\left(g_{\beta_{n}}\right)\right\rangle$. Set $a_{n i}^{\prime}:=\operatorname{deg}\left(g_{i}\right)$ and $a_{n i}:=v_{\mathbb{F}_{n-1}}\left(g_{i}\right)$. Define now the graded free $R$-module

$$
F_{n}=\bigoplus_{i=1}^{\beta_{n}} R\left(-a_{n i}^{\prime}\right)
$$


equipped with the special filtration $\mathbb{F}_{n}$ defined by the integers $v_{\mathbb{F}_{n-1}}\left(g_{i}\right)$ for $i=1, \ldots, \beta_{n}$, and let

$$
d_{n}: F_{n} \rightarrow F_{n-1}
$$

be defined by $d_{n}\left(e_{n i}\right)=g_{i}$. Consider the graded free $R$-module

$$
G_{n}=\bigoplus_{i=1}^{\beta_{n}} R\left(-a_{n i}\right), \quad \delta_{n}: G_{n} \longrightarrow G_{n-1}
$$

such that $\delta_{n}\left(e_{n i}\right)=g r_{\mathbb{F}_{n-1}}\left(g_{i}\right)$. Then

$$
\begin{aligned}
& F_{n} \stackrel{d_{n}}{\longrightarrow} F_{n-1} \stackrel{d_{n-1}}{\longrightarrow} F_{n-2} \\
& G_{n} \stackrel{\delta_{n}}{\longrightarrow} G_{n-1} \stackrel{\delta_{n-1}}{\longrightarrow} G_{n-2}
\end{aligned}
$$

are exact complexes, and we have $g r_{\mathbb{F}_{n}}\left(d_{n}\right)=\delta_{n}$. Since $\left\{g_{1}, \ldots, g_{\beta_{n}}\right\}$ is a minimal homogeneous standard basis of $\operatorname{ker}\left(d_{n-1}\right)$, again by Theorem 1.3, we get $\operatorname{ker}\left(\delta_{n}\right)=g r_{\mathbb{F}_{n}}\left(\operatorname{ker}\left(d_{n}\right)\right)$, and $\operatorname{ker}\left(d_{n}\right)$ admits a homogeneous standard basis with respect to $\mathbb{F}_{n}$.

Letting $g r_{\mathbb{F}_{n-1}}\left(g_{i}\right)={ }^{t}\left(c_{i 1}, \ldots, c_{i \beta_{n-1}}\right)$, there exists $j$ such that $c_{i j} \neq 0$ and

$$
a_{n i}=\operatorname{deg}\left(c_{i j}\right)+a_{n-1 j} .
$$

Let $g_{i}={ }^{t}\left(u_{i 1}, \ldots, u_{i \beta_{n-1}}\right)$. Since $g_{i}$ is a homogeneous element of $\operatorname{ker}\left(d_{n-1}\right)$ by $(4), u_{i j}=c_{i j}$ and

$$
a_{n i}^{\prime}=\operatorname{deg}\left(c_{i j}\right)+a_{n-1 j}^{\prime} .
$$

By inductive assumption, there exists $c \in \Delta_{\mathbb{M}}(M)$ such that $a_{n-1 j}^{\prime}=$ $a_{n-1 j}+c$; thus, we get

$$
a_{n i}^{\prime}=a_{n i}+c
$$

and hence $\Delta_{\mathbb{F}_{n}}\left(\operatorname{ker}\left(d_{n}\right)\right) \subseteq \Delta_{\mathbb{M}}(M)$ as required. We can repeat the inductive process on $\operatorname{ker}\left(d_{n}\right)$.

Remark 2.3. We remark that if for some integers $i, j$ we have $\operatorname{Tor}_{i}^{R}(M, k)_{j} \neq 0$, then $\left.\operatorname{Tor}_{i}^{R}\left(\operatorname{gr}_{\mathbb{M}}(M)\right), k\right)_{j-c} \neq 0$ for some $c \in$ $\Delta_{\mathbb{M}}(M)$. 
In fact, denote by $\mathbf{F}^{\text {min }}$ a minimal graded free resolution of $M$, since $\mathbf{F}^{\text {min }}$ is a direct summand of $\mathbf{F}$, we get

$$
\mathcal{D}\left(F_{i}^{\text {min }}\right) \subseteq \mathcal{D}\left(F_{i}\right) \text { for all } i \geq 0 .
$$

For more details, see also [11, 3.1]. Hence, the remark follows from Proposition 2.2 (iv). Always from Proposition 2.2 it will be useful to highlight that, for all $i \geq 0$ and each $b \in \mathcal{D}\left(G_{i}\right)$, there exist $a \in \mathcal{D}\left(F_{i}\right)$ and $c \in \Delta_{\mathbb{M}}(M)$ such that $a=b+c$.

Theorem 2.4. Let $R$ be a standard graded algebra, and let $M$ be a graded $R$-module equipped with the filtration $\mathbb{M}$. Then, for all $i \geq 0$,

$$
t_{i}(M) \leq t_{i}\left(g r_{\mathbb{M}}(M)\right)+v_{\mathbb{M}}(M) .
$$

Furthermore, if $M$ is of homogeneous type with respect to $\mathbb{M}$, then

$$
t_{i}\left(g r_{\mathbb{M}}(M)\right)+u_{\mathbb{M}}(M) \leq t_{i}(M) \leq t_{i}\left(g r_{\mathbb{M}}(M)\right)+v_{\mathbb{M}}(M) .
$$

Proof. By Proposition 2.2, there exists a filtered graded free resolution $\mathfrak{F}$ of $M$ such that $\operatorname{gr}_{\mathbb{F}}(\mathfrak{F})=\mathbf{G}$ is a graded minimal free resolution of $\operatorname{gr}_{\mathbb{M}}(M)$. Letting $i \geq 0$, we have $t_{i}(M) \in D\left(F_{i}\right)$. Hence, by Proposition 2.2 (iv) and Remark 2.3, there exist $b \in \mathcal{D}\left(G_{i}\right)$ and $c \in \Delta_{\mathbb{M}}(M)$ such that

$$
t_{i}(M)=b+c .
$$

Since $b \leq t_{i}\left(g r_{\mathbb{M}}(M)\right)$ and $c \leq v_{\mathbb{M}}(M)$, we get $t_{i}(M) \leq t_{i}\left(g r_{\mathbb{M}}(M)\right)+$ $v_{\mathbb{M}}(M)$. If $M$ is of homogeneous type, then $\mathbf{F}$ is minimal. Now again, by Remark 2.3, for every $i \geq 0$, there exists $a^{\prime} \in \mathcal{D}\left(F_{i}^{\text {min }}\right)$ and $c^{\prime} \in \Delta_{\mathbb{M}}(M)$ such that

$$
t_{i}\left(g r_{\mathbb{M}}(M)\right)=a^{\prime}-c^{\prime} .
$$

Considering the fact that $u_{\mathbb{M}}(M) \leq c^{\prime}$, we obtain

$$
t_{i}\left(g r_{\mathbb{M}}(M)\right)+u_{\mathbb{M}}(M) \leq t_{i}\left(g r_{\mathbb{M}}(M)\right)+c^{\prime}=a^{\prime} \leq t_{i}(M) .
$$

Corollary 2.5. With the above notation and assumptions, we have

$$
\operatorname{reg}_{R}(M) \leq \operatorname{reg}_{R}\left(g r_{\mathbb{M}}(M)\right)+v_{\mathbb{M}}(M) .
$$


Moreover, if $M$ is of homogeneous type with respect to $\mathbb{M}$, then

(8) $\operatorname{reg}_{R}\left(g r_{\mathbb{M}}(M)\right)+u_{\mathbb{M}}\left(M \leq \operatorname{reg}_{R}(M) \leq \operatorname{reg}_{R}\left(g r_{\mathbb{M}}(M)\right)+v_{\mathbb{M}}(M)\right.$.

Proof. It is a direct consequence of Proposition 2.4.

Corollary 2.6. If $\operatorname{reg}_{R}\left(g r_{\mathbb{M}}(M)\right)<\infty$, then $\operatorname{reg}_{R}(M)<\infty$. Furthermore, the converse holds, provided $M$ is of homogeneous type with respect to $\mathbb{M}$.

The assumption of being of homogeneous type cannot be deleted as the following example shows.

Example 2.7. Let $k$ be a field and $R=k[X, Y] /\left(X^{3}\right)$. Let $x, y$ be the residue class of $X, Y$ in $R$, and set $\mathfrak{m}=(x, y)$. Consider the module $M$ whose minimal graded free resolution is

$$
0 \longrightarrow R(-3) \stackrel{\left(\begin{array}{l}
x^{2} \\
y^{3}
\end{array}\right)}{\longrightarrow} R(-1) \bigoplus R(0) \longrightarrow 0 .
$$

We have $M=R(-1) \bigoplus R(0) / N$ where $N=\left(x^{2}, y^{3}\right) R$. Then $M^{g} \cong$ $R \bigoplus R / N^{*}$ where $N^{*}$ is the submodule of $R \bigoplus R$ generated by the initial forms of elements of $N$ with respect to the $\mathfrak{m}$-adic filtration. One can see that $N^{*}=\left\langle\left(x^{2}, 0\right),\left(0, x y^{3}\right)\right\rangle$. The minimal graded free resolution of $M^{g}$ is

$$
\cdots \longrightarrow R^{2} \stackrel{f_{i+1}}{\longrightarrow} R^{2} \stackrel{f_{i}}{\longrightarrow} \cdots \stackrel{f_{2}}{\longrightarrow} R^{2} \stackrel{f_{1}}{\longrightarrow} R^{2} \longrightarrow 0,
$$

where

$$
\begin{aligned}
f_{1} & =\left(\begin{array}{cc}
x^{2} & 0 \\
0 & x y^{3}
\end{array}\right), \\
f_{2 k} & =\left(\begin{array}{cc}
x & 0 \\
0 & x^{2}
\end{array}\right), \\
f_{2 k+1} & =\left(\begin{array}{cc}
x^{2} & 0 \\
0 & x
\end{array}\right) \quad \text { for } k \geq 1 .
\end{aligned}
$$

Therefore, $M$ is not of homogeneous type and $\operatorname{reg}_{R}(M)<\infty$, but

$$
\operatorname{reg}_{R}\left(M^{g}\right)=+\infty \text {. }
$$


3. Linearity defect of a graded module. In this section, we establish notation, provide the definitions of linearity defect and of Koszul modules. We suggest [5], [6] and [14] for more details concerning the previous concepts. Even if this section is mainly concerned with graded standard $k$-algebras, we present the notions in the more general case of modules over local rings in view of the next section.

Let $(R, \mathfrak{m}, k)$ be a local ring. A complex of $R$-modules

$$
\mathbf{C}=\cdots \longrightarrow C_{n+1} \longrightarrow C_{n} \stackrel{\partial_{n}}{\longrightarrow} C_{n-1} \longrightarrow \cdots
$$

is said to be minimal if $\partial_{n}\left(C_{n}\right) \subseteq \mathfrak{m} C_{n-1}$. The standard filtration $\mathfrak{F}$ of a minimal complex $\mathbf{C}$ is defined by subcomplexes $\left\{\mathfrak{F}_{i} \mathbf{C}\right\}$, where $\left(\mathfrak{F}^{i} \mathbf{C}\right)_{n}=\mathfrak{m}^{i-n} C_{n}$ for all $n \in \mathbb{Z}$. with $\mathfrak{m}^{j}=0$ for $j \leq 0$. The associated graded complex with respect to this filtration is denoted by $\operatorname{lin}^{R}(\mathbf{C})$ and called the linear part of $\mathbf{C}$. By construction, $\operatorname{lin}^{R}(\mathbf{C})$ is a minimal complex of graded modules over the graded ring $R^{g}$, and it has the property that $\operatorname{lin}^{R}(\mathbf{C})_{n}=C_{n}^{g}(-n)$.

Definition 3.1. An $R$-module $M$ is said to be $\operatorname{Koszul}$ if $\operatorname{lin}^{R}(\mathfrak{F})$ is acyclic, where $\mathfrak{F}$ is a minimal free resolution of $M$.

Herzog and Iyengar [6, 1.7] introduced an invariant which is a measure of how far $M$ is from being Koszul.

Definition 3.2. Let $M$ be an $R$-module and $\mathfrak{F}$ its minimal free resolution. Define

$$
\operatorname{ld}_{R}(M):=\sup \left\{i \in \mathbb{Z} \mid \mathrm{H}_{i}\left(\operatorname{lin}^{R}(\mathfrak{F}) \neq 0\right\},\right.
$$

and we say that $\operatorname{ld}_{R}(M)$ is the linearity defect of $M$.

By the uniqueness of a minimal free resolution up to isomorphism of complexes, one has that $\operatorname{ld}_{R}(M)$ does not depend on $\mathfrak{F}$, but only on the module $M$. It follows by the definitions that $M$ is a Koszul $R$-module if and only if $\operatorname{ld}_{R}(M)=0$. Note that, for any integer $d$, one has $\operatorname{ld}_{R}(M) \leq d$ if and only if the $d$ th syzygy module $\Omega^{d}(M)$ of $M$ is Koszul.

The next result provides a characterization of Koszul modules, see $[6,1.5]$. 
Proposition 3.3. The following facts are equivalent:

(1) $M$ is Koszul,

(2) $\operatorname{ld}_{R}(M)=0$,

(3) $\operatorname{lin}(\mathfrak{F})$ is a minimal free resolution of $M^{g}$ as a $R^{g}$-module,

(4) $M^{g}$ has a linear resolution as a $R^{g}$-module.

Note that, if $M$ is Koszul, then $M^{g}$ has a linear resolution; hence $M$ is of homogeneous type, that is, $\beta_{j}^{R}(M)=\beta_{j}^{R^{g}}\left(M^{g}\right)$ for every $j$.

Let $R$ be a graded standard $k$-algebra. If $M$ is a finitely generated graded $R$-module, one can define in the same manner the linearity defect $\operatorname{ld}_{R}(M)$ by using a minimal graded free resolution $\mathfrak{F}$ of $M$ over $R$. Notice that the complex $\operatorname{lin}^{R}(\mathfrak{F})$ is obtained from $\mathfrak{F}$ by replacing with 0 all entries of degree $>1$ in the matrices representing the homomorphisms. If a graded $R$-module $M$ has a linear resolution, then it is Koszul, but the converse fails in general; see [6, 1.9].

Accordingly, with the classical definition given by Priddy, $R$ is a Koszul graded $k$-algebra if $k$ has linear resolution as $R$-module. Because $k^{g}=k$, then by the previous proposition, $R$ is a Koszul graded $k$ algebra if and only if $k$ is a Koszul $R$-module. If $R$ is a Koszul graded algebra, it was proved by Iyengar and Römer that a graded $R$-module $M$ is Koszul if and only if $M$ is componentwise linear.

Koszul modules have a special graded free resolution. The following proposition describes the graded resolutions of Koszul modules extending to any graded standard algebra the result proved by Rossi and Sharifan in $[\mathbf{1 0}, 2.2$.$] .$

Proposition 3.4. Let $R$ be a standard graded algebra, and let $M$ be a finitely generated graded $R$-module with $\mathcal{D}(M)=\left\{i_{1}, \ldots, i_{s}\right\}$. Assume that $M$ is Koszul. Then, for each $n \geq 1$, we have

$$
\operatorname{Tor}_{n}^{R}(M, k)_{j}=0 \quad \text { for } j \neq i_{1}+n, \ldots, i_{s}+n .
$$

Furthermore, if for some $n \geq 1$ and $1 \leq r \leq s$, we have $\operatorname{Tor}_{n}^{R}(M, k)_{i_{r}+n}$ $=0$, then $\operatorname{Tor}_{m}^{R}(M, k)_{i_{r}+m}=0$, for all $m \geq n$.

Proof. Since $M$ is Koszul, then $M$ is of homogeneous type. We obtain the result as a consequence of Proposition 2.2. In fact, let $\mathfrak{F}$ 
and $\mathbf{G}$ be, respectively, minimal graded free resolutions of $M$ and $M^{g}$ as $R$-modules. Observe that $\operatorname{Tor}_{i}^{R}(M, k)_{j}=0$ if and only if $j \notin \mathcal{D}\left(F_{i}\right)$. By the assumption $\mathbf{G}$ is linear, then by Remark 2.3 we get

$$
\mathcal{D}\left(F_{n}\right) \subseteq\left\{n+i_{1}, \cdots, n+i_{s}\right\} \text { for all } n \geq 1 .
$$

Let $n \geq 1$, and assume $i_{r}+n+1 \in \mathcal{D}\left(F_{n+1}\right)$. Again since $\mathbf{G}$ is linear, we conclude that there exists $a \in \mathcal{D}\left(F_{n}\right)$ such that $i_{r}+n+1=1+a$. Hence, we get $i_{r}+n \in \mathcal{D}\left(F_{n}\right)$, and the conclusion follows inductively.

It is shown in [6] that if $\operatorname{ld}_{R}(M)$ is finite, then $\operatorname{reg}_{R}(M)$ is finite as well.

We can refine this result giving a different proof and more precise information on the regularity.

Proposition 3.5. Let $R$ be a standard graded algebra, and let $M$ be a finitely generated graded $R$-module. If $\operatorname{ld}_{R}(M)=d<\infty$, then

$$
\operatorname{reg}_{R}(M)=\max \left\{t_{i}(M)-i: 0 \leq i \leq d\right\} .
$$

In particular, if $M$ is Koszul, then $\operatorname{reg}_{R}(M)=t_{0}(M)$.

Proof. If $\operatorname{ld}_{R}(M)=0$, that is $M$ is Koszul, we have $\operatorname{reg}_{R}\left(g r_{m}(M)\right)=$ 0 . Hence, by Corollary 2.5, we conclude that $\operatorname{reg}_{R}(M) \leq t_{0}(M)$. By the fact that $t_{0}(M) \leq \operatorname{reg}_{R}(M)$, we get $\operatorname{reg}_{R}(M)=t_{0}(M)$. Now letting $\operatorname{ld}_{R}(M)=d$, the $d$ th syzygy module $\Omega^{d}(M)$ is Koszul and, by the above, $\operatorname{reg}_{R}\left(\Omega^{d}(M)\right)=t_{d}(M)$. This follows

$$
t_{i}(M)-i \leq t_{d}(M)-d \text { for all } i \geq d
$$

and then

$$
\operatorname{reg}_{R}(M)=\max \left\{t_{i}(M)-i: 0 \leq i \leq d .\right\}
$$

4. Linearity defect of a module over a local ring. Let $(R, m)$ be a local ring, and let $M$ be a finitely generated $R$-module equipped with the filtration $\mathbb{M}$. Motivated by the questions raised in the introduction, our aim is to investigate the interplay between $\operatorname{ld}_{R}(M)$ and $\operatorname{ld}_{R^{g}}\left(g r_{\mathbb{M}}(M)\right)$.

Recall that, if $R$ is a regular local ring, Rossi and Sharifan in [10, 3.6.] proved that $\operatorname{ld}_{R^{g}}\left(g r_{\mathbb{M}}(M)\right)=0$ implies $\operatorname{ld}_{R}(M)=0$, provided $M$ is minimally generated by a standard basis with respect to $\mathbb{M}$. Notice 
that Proposition 3.4 allows us to extend this result to any local ring by repeating verbatim the same proof in [10]. All the details can be found in the Ph.D. thesis of the second author, see [1].

Observe that, by Proposition 3.3, $\operatorname{ld}_{R}(M)=0$ implies $\operatorname{ld}_{R^{g}}\left(M^{g}\right)=0$; hence, $M$ is Koszul if and only if $M^{g}$ is Koszul.

Unfortunately, this statement cannot be extended to $\operatorname{ld}_{R}(M)=d$. Example 2.7 shows that $\operatorname{ld}_{R}(M)<\infty$ does not imply $\operatorname{ld}_{R^{g}}\left(M^{g}\right)<\infty$. In fact, there exists a module such that $\operatorname{ld}_{R}(M)<\infty$ has finite homological dimension, but $\operatorname{ld}_{R^{g}}\left(M^{g}\right)$ is infinite since the regularity is infinite, see Proposition 3.5.

Because our aim would be to give an answer to Question 1 based on $k$ as an $R$-module, one can ask Question 2 for cyclic modules. The next example disproves the assertion.

Example 4.1. Consider $R=k[[x, y, z, u]] /\left(x^{3}\right)$, and let $J=\left(x^{3}, y^{2}+\right.$ $\left.x^{2}, z^{2} y+u^{4}\right) /\left(x^{3}\right)$. The ideal $J$ is generated by a complete intersection; hence, the homological dimension of $R / J$ as an $R$-module is finite and so the linearity defect is finite.

Note that $R^{g}$ is a hypersurface and, $\operatorname{since} \operatorname{depth}\left(R^{g}\right)-\operatorname{depth}\left((R / J)^{g}\right)=$ 3 by [2, Theorem 5.1.1], the minimal graded $R^{g}$-free resolution of $(R / J)^{g}$ becomes periodic of period 2 and $\beta_{i}\left((R / J)^{g}\right)=\beta_{i+1}\left((R / J)^{g}\right)$ for $i \geq 3$. By computing the third and fourth syzgies of $(R / J)^{g}$ we deduce that $\operatorname{reg}_{R^{g}}\left((R / J)^{g}\right)$ is infinite; therefore, $\operatorname{ld}\left((R / J)^{g}\right)$ is infinite.

We will prove that, if $M$ is of homogeneous type, the finiteness of $\operatorname{ld}_{R}(M)$ controls the regularity of $g r_{\mathbb{M}}(M)$. Notice that finiteness of the regularity of a graded module does not imply finiteness of the linearity defect.

We need a technical lemma. With the notation fixed in Proposition 2.2 , let $\left(\mathbf{G}, \delta\right.$.) be the minimal graded $R^{g}$-free resolution of $g r_{\mathbb{M}}(M)$. We build up an $R$-free resolution $(\mathfrak{F}, d$.) of $M$ and denote by $\mathcal{M}_{n}=\left(m_{r s}\right)$ the corresponding matrix associated to the differential map $d_{n}: F_{n} \rightarrow F_{n-1}$.

Lemma 4.2. With the above notations if, for all $n \geq 1$, the matrix 
$\mathcal{M}_{n}$ has an entry of $\mathfrak{m}$-adic valuation $\leq 1$ in each column, then

$$
\operatorname{reg}_{R^{g}}\left(g r_{\mathbb{M}}(M)\right)=t_{0}\left(g r_{\mathbb{M}}(M)\right) .
$$

Proof. Let

$$
\mathbf{G} .=\cdots \longrightarrow \bigoplus_{j=1}^{\beta_{1}} R^{g}\left(-a_{1 j}\right) \stackrel{\delta_{1}}{\longrightarrow} \bigoplus_{j=1}^{\beta_{0}} R^{g}\left(-a_{0 j}\right) \stackrel{\delta_{0}}{\longrightarrow} g r_{\mathbb{M}}(M) \longrightarrow 0
$$

be the minimal graded free resolution of $\operatorname{gr}_{\mathbb{M}}(M)$, and let $U_{j}=\left(u_{r s}\right)$ be the $j$-th degree matrix of $g r_{\mathbb{M}}(M)$, where

$$
u_{r s}=a_{j s}-a_{j-1 r} .
$$

We denote by $\mathcal{M}_{j}^{*}=\left(n_{r s}\right)$ the corresponding matrix associated to $\delta_{j}$. By Proposition 2.2 (ii) and (iii), the columns of $\mathcal{M}_{j}^{*}$ are the initial forms of the corresponding columns of $\mathcal{M}_{j}$ with respect to the special filtration which has been defined on $F_{j-1}$. In particular, the degree matrix $U_{j}$ controls the valuations of entries of $\mathcal{M}_{j}$ with respect to the $\mathfrak{m}$-adic filtration. We have

$$
\begin{cases}v_{R}\left(m_{r s}\right)=u_{r s} & \text { if } n_{r s} \neq 0 \\ v_{R}\left(m_{r s}\right)>u_{r s} & \text { otherwise }\end{cases}
$$

We prove by induction on $j \geq 0$ that, for every $j$ and $1 \leq s \leq \beta_{j}$, there exists $i, 1 \leq i \leq \beta_{0}$ such that $a_{j s} \leq j+a_{0 i}$. The case $j=0$ is obvious. Let $j>0$, and suppose that the result has been proved for every $0 \leq i<j$. Let $1 \leq s \leq \beta_{j}$, by the assumption there exists $r$ with $1 \leq r \leq \beta_{j-1}$ such that for the entry $m_{r s}$ of $\mathcal{M}_{j-1}$, we have $v_{R}\left(m_{r s}\right) \leq 1$. If $v_{R}\left(m_{r s}\right)=0$, then $n_{r s}=0$, since $\mathbf{G}$. is minimal. Then, from (9) we get $a_{j s}<a_{j-1 r}$. By inductive assumption, there exists $i, 1 \leq i \leq \beta_{0}$ such that $a_{j-1 r} \leq j-1+a_{0 i}$. Thus, we conclude $a_{j s} \leq j+a_{0 i}$. If $v_{R}\left(m_{r s}\right)=1$, by (9) we may have two cases

$$
\left\{\begin{array}{l}
a_{j s}=a_{n-1 r}+1 \\
a_{j-1 r}+1>a_{j s}
\end{array}\right.
$$

By inductive assumption in each case we get

$$
a_{j s} \leq j+a_{0 i} \text { for some } 1 \leq i \leq \beta_{0} .
$$


Therefore, we conclude that for each $j>0$ there exists $i, 1 \leq i \leq \beta_{0}$ such that $t_{j}\left(g r_{\mathbb{M}}(M)\right) \leq j+a_{0 i}$. This holds that

$$
t_{j}\left(g r_{\mathbb{M}}(M)\right) \leq j+t_{0}\left(g r_{\mathbb{M}}(M)\right),
$$

and hence

$$
\operatorname{reg}_{R^{g}}\left(g r_{\mathbb{M}}(M)\right)=t_{0}\left(g r_{\mathbb{M}}(M)\right) .
$$

Theorem 4.3. Let $(R, m)$ be a local ring, and let $M$ be a Koszul module equipped with the filtration $\mathbb{M}$. If $M$ is of homogeneous type with respect to $\mathbb{M}$, then

$$
\operatorname{reg}_{R^{g}}\left(g r_{\mathbb{M}}(M)\right)=t_{0}\left(g r_{\mathbb{M}}(M)\right) .
$$

Proof. Let $\mathbf{G}$ be the minimal graded $R^{g}$-free resolution of $g r_{\mathbb{M}}(M)$. Since $M$ is of homogeneous type, then the resolution $\mathbf{F}$ is minimal. By assumption, $\operatorname{lin}(\mathbf{F})$ is acyclic; therefore, each column of the matrices associated to the differential maps of $\operatorname{lin}(\mathbf{F})$ has a non-zero linear form. This follows that each column of the corresponding matrices of $\mathbf{F}$ has an entry of valuation one with respect to the m-adic filtration. Now the conclusion follows from Lemma 4.2.

Corollary 4.4. Let $(R, \mathfrak{m})$ be a local ring, and let $M$ be a finitely generated $R$-module equipped with the filtration $\mathbb{M}$. Let $\operatorname{ld}_{R}(M)=d<$ $\infty$, and assume that $M$ is of homogeneous type with respect to $\mathbb{M}$, then

$$
\operatorname{reg}_{R^{g}}\left(g r_{\mathbb{M}}(M)\right)=\max \left\{t_{i}\left(g r_{\mathbb{M}}(M)\right)-i ; 0 \leq i \leq d\right\} .
$$

Proof. Since $\operatorname{ld}_{R}(M)=d$, then the $d$ th syzygy $\Omega^{d}(M)$ of $M$ is Koszul. Hence, we may apply Theorem 4.3, and we conclude that $\operatorname{reg}_{R^{g}}\left(g r_{\mathbb{F}}\left(\Omega^{d}(M)\right)\right)=t_{0}\left(g r_{\mathbb{F}}\left(\Omega^{d}(M)\right)\right.$. We proceed now as in the proof of Proposition 3.5.

We are ready now to give a partial answer to Question 1 .

Proposition 4.5. Let $(R, \mathfrak{m}, k)$ be a local ring. If $\operatorname{ld}_{R}(k)<\infty$ and $k$ is of homogeneous type, then $\operatorname{ld}_{R}(k)=0$.

Proof. By Corollary 4.4, we get $\operatorname{reg}_{R^{g}}(k)<\infty$, then according to Avramov and Peeva [4, Theorem 2] we obtain $\operatorname{ld}_{R}(k)=0$. 


\section{REFERENCES}

1. R. Ahangari Maleki, Koszul rings and modules and Castelnuovo-Mumford regularity, Ph.D. thesis, Kharazmi University, Iran, 2013.

2. L.L. Avramov, Infinite free resolutions, six lectures on commutative algebra (Bellaterra, 1996). Progr. Math. 166, Birkhauser, Basel, 1998, 1-118.

3. L.L. Avramov and D. Eisenbud, Regularity of modules over a Koszul algebra, J. Alg. 153 (1992), 85-90.

4. L.L. Avramov and I. Peeva, Finite regularity and Koszul algebras, Amer. J. Math. 123 (2001), 275-281.

5. A. Conca, E. De Negri and M.E. Rossi, Koszul algebras and regularity, Irena Peeva ed., Commutative algebra: Expository papers dedicated to David Eisenbud, Springer, New York,

6. J. Herzog and S. Iyengar, Koszul modules, J. Pure Appl. Alg. 201 (2005), 154188.

7. J. Herzog, M.E. Rossi and G. Valla, On the depth of the symmetric algebra, Trans. Amer. Math. Soc. 296 (1986), 577-606.

8. S. Iyengar and T. Römer, Linearity defects of modules over commutative rings, J. Alg. 322 (2009), 3212-3237.

9. L. Robbiano and G. Valla, Free resolutions for special tangent cones, Comm. Alg. Lect. Notes Pure Appl. Math. 84, Dekker, New York, 1983.

10. M.E. Rossi and L. Sharifan, Minimal free resolution of a finitely generated module over a regular local ring, J. Alg. 322 (2009), 3693-3712.

11. Consecutive cancellation in Betti numbers of local rings, Proc. Amer. Math. Soc. 138 (2010), 61-73.

12. M.E. Rossi and G. Valla, Hilbert functions of filtered modules, Lect. Notes Un. Matem. Ital. 9, Springer-Verlag, Berlin; UMI, Bologna, 2010.

13. L.M. Şega, On the linearity defect of the residue field, arXiv:1303.4680v1 [math.AC], 2013.

14 . Homological properties of powers of the maximal ideal of a local ring, J. Alg. 241 (2001), 827-858.

15. T. Shibuta, Cohen-Macaulyness of almost complete intersection tangent cones, J. Alg. 319 (2008), 3222-3243.

School of Mathematics, Institute for Research in Fundamental Sciences (IPM), P.O. Box 19395-5746, Tehran, Iran

Email address: rasoulahangari@gmail.com, rahangari@ipm.ir

Department of Mathematics, University of Genova, Via Dodecaneso 35 , 16146-Genova, Italy

Email address: rossim@dima.unige.it 